\title{
A Modified Fuzzy C-Means Algorithm for MRI Bias Field Estimation and Adaptive Segmentation
}

\author{
M. N. Ahmed, S. M. Yamany, N. A. Mohamed, and A. A. Farag \\ Computer Vision and Image Processing Lab \\ Univ. of Louisville, E.E. Dept., Louisville, KY 40292 USA
}

\begin{abstract}
In this paper, we present a novel algorithm for adaptive fuzzy segmentation of MRI data and estimation of intensity inhomogeneities using fuzzy logic. MRI intensity inhomogeneities can be attributed to imperfections in the RF coils or some problems associated with the acquisition sequences. The result is a slowly-varying shading artifact over the image that can produce errors with conventional intensity-based classification. Our algorithm is formulated by modifying the objective function of the standard fuzzy c-means (FCM) algorithm to compensate for such inhomogeneities and to allow the labeling of a pixel (voxel) to be influenced by the labels in its immediate neighborhood. The neighborhood effect acts as a regularizer and biases the solution towards piecewisehomogeneous labeling. Such a regularization is useful in segmenting scans corrupted by salt and pepper noise. Experimental results on both synthetic images and MR data are given to demonstrate the effectiveness and efficiency of the proposed algorithm.
\end{abstract}

\section{Introduction}

Spatial intensity inhomogeneity induced by the radio frequency $(\mathrm{RF})$ coil in magnetic resonance imaging (MRI) is a major problem in the computer analysis of MRI data. Such inhomogeneities have rendered conventional intensity-based classification of MR images very difficult, even with advanced techniques such as non-parametric, multichannel methods.[1,2]. This is due to the fact that the intensity inhomogeneities appearing in MR images produce spatial changes in tissue statistics, i.e. mean and variance. In addition, the degradation on the images obstructs the physician's diagnoses because the physician has to ignore the inhomogeneity artifact in the corrupted images [3].

The removal of the spatial intensity inhomogeneity from MR images is difficult because the inhomogeneities could change with different MRI acquisition parameters from patient to patient and from slice to slice. Therefore, the correction of intensity inhomogeneities is usually required for each new image. In the last decade, a number of algorithms have been proposed for the intensity inhomogeneity correction. Meyer et al.[5] presented an edge-based segmentation scheme to find uniform regions in the image followed by a polynomial surface fit 
to those regions. The result of their correction is, however, very dependent on the quality of the segmentation step.

Several authors have reported methods based on the use of phantoms for intensity calibration. Wicks et al.[4] proposed methods based on the signal produced by a uniform phantom to correct for MRI images of any orientation. Similarly, Tincher et al.[6] modeled the inhomogeneity function by a secondorder polynomial and fitted it to a uniform phantom-scanned MR image. These phantom approaches, however, have the drawback that the geometry relationship of the coils and the image data is typically not available with the image data. They also require the same acquisition parameters for the phantom scan and the patient. In addition, these approaches assume the intensity corruption effects are the same for different patients, an assumption which is not valid, in general [3].

The homomorphic filtering approach to remove the multiplicative effect of the inhomogeneity has been commonly used because of its easy and efficient implementation [2]. This method, however, is effective only on images with relatively low contrast. Some researchers $[6,7]$ reported undesirable artifacts.

Dawant et al.[7] used operator-selected reference points in the image to guide the construction of a thin-plate spline correction surface. The performance of this method substantially depends on the labeling of the reference points. Considerable user interactions are usually required to obtain good correction results. More recently, Gilles et al.[8] proposed an automatic and iterative B-spline fitting algorithm for the intensity inhomogeneity correction of breast MR images. The application of this algorithm is restricted to MR images with a single dominant tissue class, such as the breast MR images. Another polynomial surface fitting method [9] was proposed based on the assumption that the number of tissue classes, the true means, and standard deviations of all the tissue classes in the image are given. Unfortunately, the required statistical information is usually not available.

A different approach to segment images with intensity inhomogeneities is to simultaneously compensate for the shading effect while segmenting the image. This has the advantage of being able to use intermediate information from the segmentation while performing the correction. Recently, Wells et al.[1] developed a new statistical approach based on the EM algorithm to solve the bias field correction problem and the tissue classification problem altogether in an alternative fashion. Guillemaud and Brady [10] further refined this technique by introducing an extra class, "other". There are two main disadvantages of this EM approach. First, the the EM algorithm is extremely computationally intensive, especially for large problems. Second, the EM algorithm requires a good initial guess for either the bias field or for the classification estimate. Otherwise, the EM algorithm could be easily trapped in a local minimum, resulting in an unsatisfactory solution [3]. Xu et al.[11] followed Wells' approach and proposed a new adaptive fuzzy c-means technique to produce fuzzy segmentation while compensating for intensity inhomogeneities. Their method, however, is computationally intensive and is also very sensitive to noise. 
In this paper, we present a different approach for adaptive fuzzy segmentation of MRI data. Our algorithm is formulated by modifying the objective function of the standard fuzzy c-means (FCM) algorithm to compensate for such inhomogeneities and to allow the labeling of a pixel (voxel) to be influenced by the labels in its immediate neighborhood. Taking into account the neighborhood effect acts as a regularizer and biases the solution towards piecewise-homogeneous labeling. Such a regularization is useful in segmenting scans corrupted by salt and pepper noise.

\section{Background}

The observed MRI signal is modeled as a product of the true signal generated by the underlying anatomy, and a spatially-varying factor called the gain field.

$$
Y_{k}=X_{k} G_{k} \quad \forall k \in\{1,2, . ., N\}
$$

where $X_{k}$ and $Y_{k}$ are the corrected and observed intensities at the $k$ th voxel, respectively, $G_{k}$ is the gain field at the $k$ th voxel, and $N$ is the total number of voxels in the MRI volume. The application of a logarithmic transformation to the intensities allows the artifact to be modeled as an additive bias field [1]

$$
y_{k}=x_{k}+\beta_{k} \quad \forall k \in\{1,2, . ., N\}
$$

where $x_{k}$ and $y_{k}$ are the corrected and observed log-transformed intensities at the $k$ th voxel, respectively, and $\beta_{k}$ is the bias field at the $k$ th voxel. If the gain field is known, then it is relatively easy to estimate the tissue class by applying a conventional intensity-based segmentation to the corrected data. Similarly, if the tissue classes are known, then we can estimate the gain field. It may be problematic to estimate either without the knowledge of the other. We will show that by employing an iterative algorithm based on fuzzy logic, we can estimate both.

\section{Modified Fuzzy C-means (MFCM) Objective Function}

The standard FCM objective function for partitioning $\left\{x_{k}\right\}_{k=1}^{N}$ into $c$ clusters is given by

$$
J=\sum_{i=1}^{c} \sum_{k=1}^{N} u_{i k}^{p}\left\|x_{k}-v_{i}\right\|^{2}
$$

where $\left\{v_{i}\right\}_{i=1}^{c}$ are the prototypes of the clusters and the array $\left[u_{i k}\right]=U$ represents a partition matrix, $U \in \mathcal{U}$, namely

$$
\mathcal{U}\left\{u_{i k} \in[0,1] \mid \sum_{i=1}^{c} u_{i k}=1 \quad \forall k \quad \text { and } \quad 0<\sum_{k=1}^{N} u_{i k}<N \quad \forall i\right\}
$$


The parameter $p$ is a weighting exponent on each fuzzy membership and determines the amount of fuzziness of the resulting classification. The FCM objective function is minimized when high membership values are assigned to voxels whose intensities are close to the centroid of its particular class, and low membership values are assigned when the voxel data is far from the centroid. We propose to modify Eq.(3) by introducing a term that allow the labeling of a pixel (voxel) to be influenced by the labels in its immediate neighborhood. As mentioned before, the neighborhood effect acts as a regularizer and biases the solution towards piecewise-homogeneous labeling. Such a regularization is useful in segmenting scans corrupted by salt and pepper noise. The modified objective function is given by

$$
J_{m}=\sum_{i=1}^{c} \sum_{k=1}^{N} u_{i k}^{p}\left\|x_{k}-v_{i}\right\|^{2}+\frac{\alpha}{N_{R}} \sum_{i=1}^{c} \sum_{k=1}^{N} u_{i k}^{p}\left(\sum_{x_{r} \in \mathcal{N}_{k}}\left\|x_{r}-v_{i}\right\|^{2}\right)
$$

where $\mathcal{N}_{k}$ stands for the set of neighbors of $x_{k}$ and $N_{R}$ is the cardinality of $\mathcal{N}_{k}$. The neighbors effect term is controlled by the parameter $\alpha$. The relative importance of the regularizing term is inversely proportional to the signal to noise ratio (SNR) of MRI signal. Lower SNR would require higher value of the parameter $\alpha$. Substituting Eq.(2) into Eq.(5), we have

$$
J_{m}=\sum_{i=1}^{c} \sum_{k=1}^{N} u_{i k}^{p}\left\|y_{k}-\beta_{k}-v_{i}\right\|^{2}+\frac{\alpha}{N_{R}} \sum_{i=1}^{c} \sum_{k=1}^{N} u_{i k}^{p}\left(\sum_{y_{r} \in \mathcal{N}_{k}}\left\|y_{r}-\beta_{r}-v_{i}\right\|^{2}\right)
$$

Formally, the optimization problem becomes

$$
\min _{U,\left\{v_{i}\right\}_{i=1}^{c},\left\{\beta_{k}\right\}_{k=1}^{N}} J_{m} \quad \text { subject to } \quad U \in \mathcal{U}
$$

\section{Parameter Estimation}

The objective function $J_{m}$ can be minimized in a fashion similar to the standard FCM algorithm. Taking the first derivatives of $J_{m}$ with respect to $u_{i k}, v_{i}$, and $\beta_{k}$ and setting them to zero results in three conditions for $J_{m}$ to be at a minimum. In the following subsections, we will derive these three conditions.

\subsection{Membership Evaluation}

The constrained optimization in equation (7) will be solved using Lagrange multipliers

$$
J_{m}=\sum_{i=1}^{c} \sum_{k=1}^{N}\left(u_{i k}^{p} D_{i k}+\frac{\alpha}{N_{R}} u_{i k}^{p} \gamma_{i}\right)+\lambda\left(1-\sum_{i=1}^{c} u_{i k}\right)
$$


where $D_{i k}=\left\|y_{k}-\beta_{k}-v_{i}\right\|^{2}$ and $\gamma_{i}=\left(\sum_{y_{r} \in \mathcal{N}_{k}}\left\|y_{r}-\beta_{r}-v_{i}\right\|^{2}\right)$. Taking the derivative of $J_{m}$ w.r.t. $u_{i k}$ and setting the result to zero, we have

$$
\left[\frac{\delta J_{m}}{\delta u_{i k}}=p u_{i k}^{p-1} D_{i k}+\frac{\alpha p}{N_{R}} u_{i k}^{p} \gamma_{i}-\lambda\right]_{u_{i k}=u_{i k}^{*}}=0
$$

Solving for $u_{i k}$ we have

$$
u_{i k}^{*}=\left(\frac{\lambda}{p\left(D_{i k}+\frac{\alpha}{N_{R}} \gamma_{i}\right)}\right)^{\frac{1}{p-1}}
$$

Since $\sum_{j=1}^{c} u_{j k}=1 \quad \forall k$, then

$$
\sum_{j=1}^{c}\left(\frac{\lambda}{p\left(D_{j k}+\frac{\alpha}{N_{R}} \gamma_{j}\right)}\right)^{\frac{1}{p-1}}=1
$$

or

$$
\lambda=\frac{p}{\left(\sum_{j=1}^{c}\left(\frac{1}{\left(D_{j k}+\frac{\alpha}{N_{R}} \gamma_{j}\right)}\right)^{\frac{1}{p-1}}\right)^{p-1}}
$$

Substituting into equation (10), the zero-gradient condition for the membership estimator can be rewritten as,

$$
u_{i k}^{*}=\frac{1}{\sum_{j=1}^{c}\left(\frac{D_{i k}+\frac{\alpha}{N_{R}} \gamma_{i}}{D_{j k}+\frac{\alpha}{N_{R}} \gamma_{j}}\right)^{\frac{1}{p-1}}}
$$

\subsection{Cluster Prototype Updating}

In the following derivation we use the standard Eucledian distance. Taking the derivative of $J_{m}$ w.r.t. $v_{i}$ and setting the result to zero we have;

$$
\left[\sum_{k=1}^{N} u_{i k}^{p}\left(y_{k}-\beta_{k}-v_{i}\right)+\sum_{k=1}^{N} u_{i k}^{p} \frac{\alpha}{N_{R}} \sum_{y_{r} \in \mathcal{N}_{k}}\left(y_{r}-\beta_{r}-v_{i}\right)\right]_{v_{i}=v_{i}^{*}}=0
$$

Solving for $v_{i}$ we have;

$$
v_{i}^{*}=\frac{\sum_{k=1}^{N} u_{i k}^{p}\left(\left(y_{k}-\beta_{k}\right)+\frac{\alpha}{N_{R}} \sum_{y_{r} \in \mathcal{N}_{k}}\left(y_{r}-\beta_{r}\right)\right)}{(1+\alpha) \sum_{k=1}^{N} u_{i k}^{p}}
$$




\subsection{Bias Field Estimation}

In a similar fashion, taking the derivative of $J_{m}$ w.r.t $\beta_{k}$ and setting the result to zero we have

$$
\left[\sum_{i=1}^{c} \frac{\partial}{\partial \beta_{k}} \sum_{k=1}^{N} u_{i k}^{p}\left(y_{k}-\beta_{k}-v_{i}\right)^{2}\right]_{\beta_{k}=\beta_{k}^{*}}=0
$$

Since only the $k$ th term in the second summation depends on $\beta_{k}$, we have

$$
\left[\sum_{i=1}^{c} \frac{\partial}{\partial \beta_{k}} u_{i k}^{p}\left(y_{k}-\beta_{k}-v_{i}\right)^{2}\right]_{\beta_{k}=\beta_{k}^{*}}=0
$$

Differentiating the distance expression, we obtain

$$
\left[y_{k} \sum_{i=1}^{c} u_{i k}^{p}-\beta_{k} \sum_{i=1}^{c} u_{i k}^{p}-\sum_{i=1}^{c} u_{i k}^{p} v_{i}\right]_{\beta_{k}=\beta_{k}^{*}}=0
$$

Thus, the zero-gradient condition for the bias field estimator is expressed as

$$
\beta_{k}^{*}=y_{k}-\frac{\sum_{i=1}^{c} u_{i k}^{p} v_{i}}{\sum_{i=1}^{c} u_{i k}^{p}}
$$

\section{MFCM Algorithm}

The MFCM algorithm for correcting the bias field and segmenting the image into different clusters can be summarized in the following steps:

Step 1 Select initial class prototypes $\left\{v_{i}\right\}_{i=1}^{c}$. Set $\left\{\beta_{k}\right\}_{k=1}^{N}$ to zero.

Step 2 Update the partition matrix using eq.13

Step 3 The prototypes of the clusters are obtained in the form of weighted averages of the patterns using Eq.15.

Step 4 Estimate the bias term using Eq.19.

Repeat steps 2-4 until convergence.

\section{Results}

In this section we describe the application of the adaptive segmentation on synthetic images corrupted with multiplicative gain and on brain MR images. All of the MR images shown in this section were obtained using a General Electric Signa 1.5 Tesla clinical MR imager. Results show that intensity variations across patients, scans, and equipment changes have been accommodated in the estimated bias field without the need for manual intervention. We implemented the MFCM algorithm on a Silicon Graphics Onyx Supercomputer. In all the examples, we set the parameter $\alpha$ (the neighbors effect) to be 0.7 . Figure 1 shows 

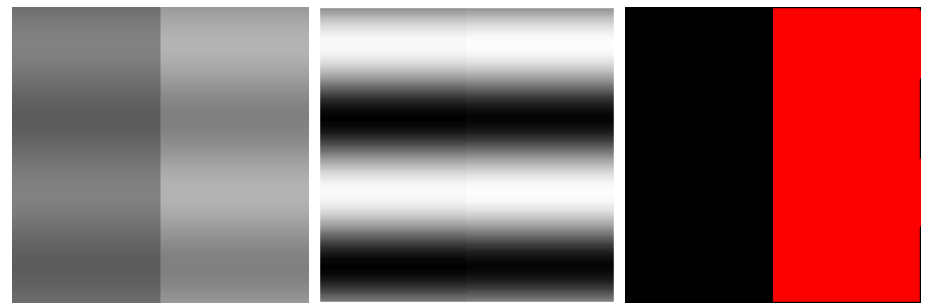

Fig. 1. Results of the MFCM on a two-class synthetic image corrupted by sinusoidal bias field. The original image is shown (left). The (Middle) presents the final results of the bias field and (right) the segmentation.

a synthetic test image. This image contains a two-class pattern corrupted by a sinusoidal gain field of higher spatial frequency. The test image is intended to represent two tissue classes, while the sinusoid represents an intensity inhomogeneity. This model was constructed so that it would be difficult to correct using homomorphic filtering or traditional FCM approaches. As shown in Figure 1, the MFCM has succeeded in correcting and classifying the data.

Figure 2 shows the results of applying the MFCM algorithm to segment an image of an axial-sectioned T1 MR brain. Strong inhomogeneities are apparent in the image. The MFCM algorithm segmented the image into three classes corresponding to background, gray matter, and white matter. The figure illustrates intensity profiles in the image before and after correction. These profiles correspond to the 128th column extract from the original bias field corrupted image and the corrected one using the MFCM algorithm. The figure demonstrates the ability of the MFCM algorithm to correct for the bias field. Figure 3 shows another example of T2 MR image where the MFCM was successful in recovering the bias field and segmenting the image. Figure 4 shows the results of applying the MFCM for the segmentation of a noisy brain image. The results of using the traditional FCM without considering the neighborhood field effect and the MFCM are presented. Notice that the segmentation of the MFCM, which uses the the neighborhood field effect, is much less fragmented compared to the traditional FCM approach. As mentioned before, the relative importance of the regularizing term is inversely proportional to the signal to noise ratio (SNR) of MRI signal.

We compared our results with the Expectation-Maximization (EM) algorithm developed by Wells et al.[1]. The MFCM algorithm produces similar results as the EM algorithm with faster convergence and the added advantage that it requires no user interaction. Correct estimates of region statistics are crucial for the convergence of the EM algorithm while in the MFCM, approximate values for class centers are sufficient. In noisy images, the MFCM technique greatly outperforms the EM algorithm as it compensates for noise by including a regularization term. While only $2 \mathrm{D}$ results were presented in this summary, the MFCM can be easily extended to 3D segmentation and bias field correction. 


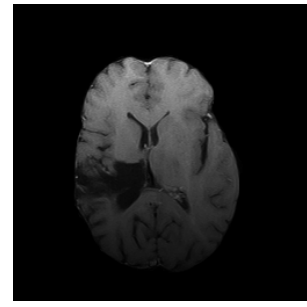

(a)

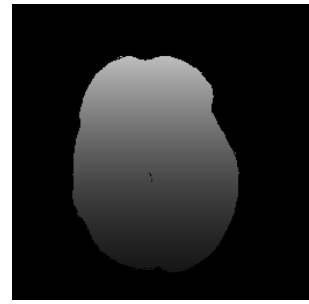

(d)

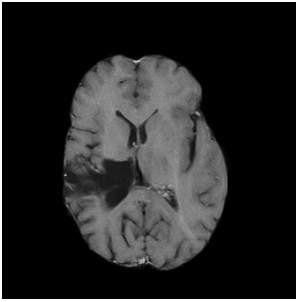

(b)

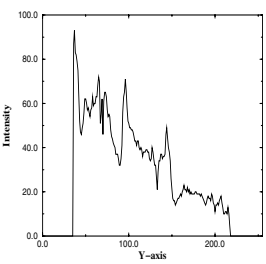

(e)

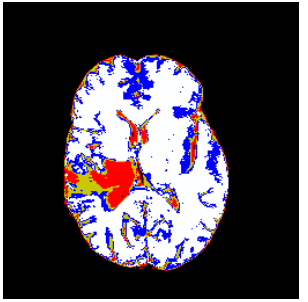

(c)

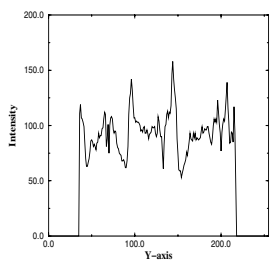

(f)

Fig. 2. Brain MRI example: (a) the original MR image corrupted with intensity inhomogeneities (b) the bias-field corrected image using the proposed algorithm. The segmented image is shown in (c) while the recovered bias field is displayed in (d). The cross section at the 128th column extract from (e) the original bias field corrupted image and (f) the corrected image using the MFCM algorithm. Intensity uniformity is clearly noticed in (f).

\section{Conclusions}

We have demonstrated a new modified fuzzy c-means (MFCM) algorithm for adaptive segmentation and intensity correction of MR images. The algorithm was formulated by modifying the objective function of the standard fuzzy cmeans (FCM) algorithm to compensate for such inhomogeneities and to allow the labeling of a pixel (voxel) to be influenced by the labels in its immediate neighborhood. Taking into account the neighborhood effect acts as a regularizer and biases the solution towards piecewise-homogeneous labeling. Such a regularization is useful in segmenting scans corrupted by salt and pepper noise. The MFCM segmentation increases the robustness and level of automation available for the segmentation of MR images into tissue classes by correcting interscan intensity inhomogeneities. Via improved segmentation, this algorithm leads to an improvement in the quality of 3D reconstruction of brain structures, and for visualization, surgical planning, disease research, and for other purposes. 


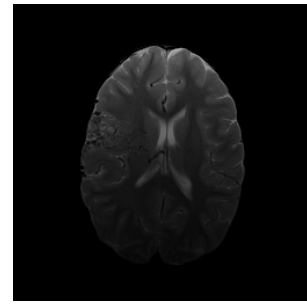

(a)

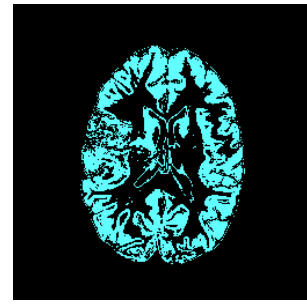

(d)

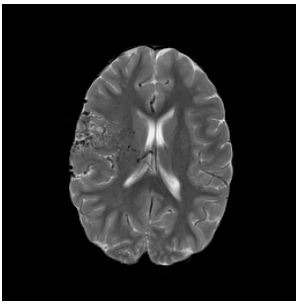

(b)

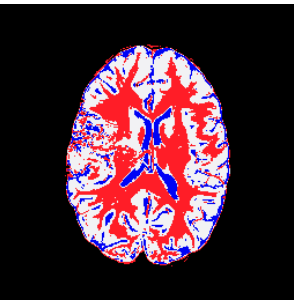

(e)

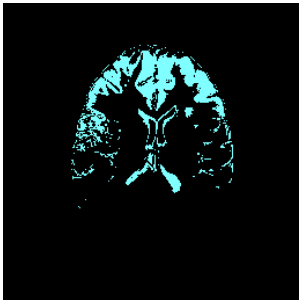

(c)

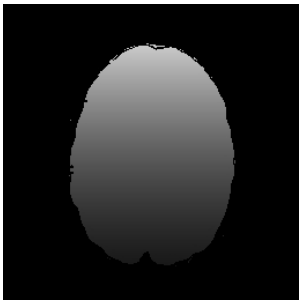

(f)

Fig. 3. Brain MRI example: (a) the original MR image corrupted with intensity inhomogeneities (b) the bias-field corrected image using the proposed MFCM algorithm. (c) Gray matter membership using traditional FCM. (d) Gray matter membership using MFCM. The segmented image using MFCM is shown in (e) while the recovered bias field is displayed in (f).

\section{References}

1. W.M. Wells, III, W.E.L. Grimson, R. Kikinis and F.A. Jolesz "Adaptive segmentation of MRI data," IEEE Trans. Med. Imag., Vol. 15, pp. 429-442, 1996.72 , $73,74,78$

2. B. Johnston, M.S. Atkins, B. Mackiewich and M. Anderson, "Segmentation of multiple sclerosis lesions in intensity corrected multispectral MRI," IEEE Trans. Medical Imaging, Vol. 15, No.2, pp. 154-169, 1996. 72, 73

3. S. Lai and M. Fang, "A new variational shape-from-orientation approach to correcting intensity inhomogeneities in MR images," Workshop on Biomedical Image Analysis, CVPR98, pp. 56-63, Sanata Barbara, California, 1998. 72, 73

4. D.A.G. Wicks, G.J. Barker and P.S. Tofts, "Correction of intensity nonuniformity in MR images of any orientation," Magnetic Resonance Imaging, Vol. 11, pp. 183-196, 1993. 73

5. C.R. Meyer, P.H. Bland and J. Pipe, "Retrospective correction of intensity inhomogeneities in MRI," IEEE Trans. Medical Imaging, Vol. 14, No. 1, pp. 36-41, 1995. 72 


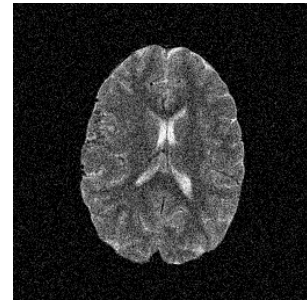

(a)

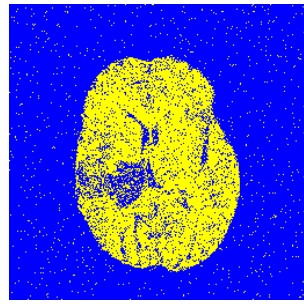

(d)

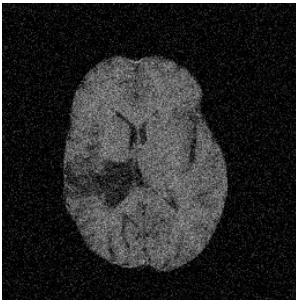

(b)

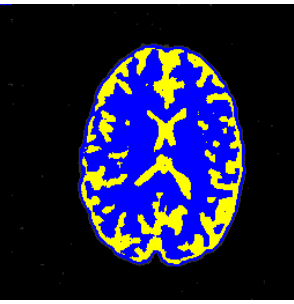

(e)

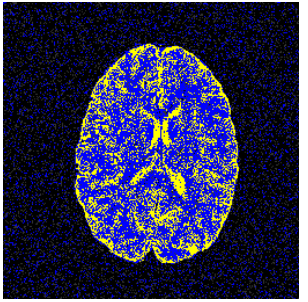

(c)

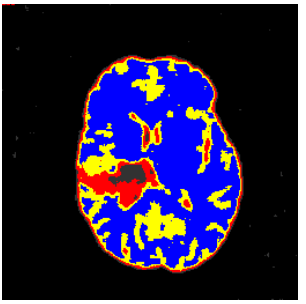

(f)

Fig. 4. Brain Tumor MRI examples corrupted with noise: The original MR images corrupted with salt and pepper noise are shown in (a) and (b). The segmented images using MFCM without any neighborhood consideration, are presented in (c) and (d). The segmented images using MFCM and neighborhood effect are presented in (e) and (f). See the text for a discussion on the results.

6. M. Tincher, C.R. Meyer, R. Gupta and D.M. Williams, "Polynomial modeling and reduction of RF body coil spatial inhomogeneity in MRI," IEEE Trans. Medical Imaging, Vol. 12, No. 2, pp. 361-365, 1993. 73

7. B. Dawant, A. Zijidenbos, and R Margolin "Correction of intensity variations in MR images for computer-aided tissue classification," IEEE Trans. Med. Imag., Vol. 12, pp. 770-781 1993. 73

8. S. Gilles, M.Brady, J. Declerck, J.P. Thirion and N. Ayache, "Bias field correction of breast MR images," Proceedings of the Fourth International Conf. on Visualization in Biomedical Computing, pp. 153-158, Hamburg, Germany, Sep. 1996. 73

9. C. Brechbuhler, G. Gerig and G. Szekely, "Compensation of spatial inhomogeneity in MRI based on a parametric bias estimate," Proceedings of the Fourth International Conference on Visualization in Biomedical Computing, pp. 141,146, Hamburg, Germany, Sep. 1996. 73

10. R. Guillemaud and M. Brady, "Estimating the bias field of MR images," IEEE Trans. Medical Imaging, Vol. 16, No. 3, pp. 238-251, 1997. 73

11. C. Xu, D. Pham, and Jerry Prince "Finding the brain cortex using fuzzy segmentation, isosurfaces, and deformable surfaces," Pro. of the XVth Int. Conf. on Information Processing in Medical Imaging (IPMI 97)., pp. 399-404, 1997. 73 